\title{
A Survey on Technologies and Challenges of LTE-U
}

\author{
Tong Gan ${ }^{1}$, Shi-You Wang ${ }^{1}$, Qiang Ma ${ }^{1}$, Yi-Dong $\mathrm{Jia}^{2, *}$ and Yun-Yun $\mathrm{Ma}^{3}$
}

\author{
${ }^{1}$ Beijing Metro Consultancy Corporation Ltd., Beijing, 100071, China \\ ${ }^{2}$ School of Computer and Communication Engineering, University of Science and Technology Beijing, Beijing, 100083, China \\ ${ }^{3}$ School of Electronic Electrical and Systems Engineering, University of Birmingham, Birmingham, B15 2TT, United Kingdom \\ *Corresponding Author: Yi-Dong Jia. Email: g20188737@xs.ustb.edu.cn \\ Received: 07 April 2021; Accepted: 01 June 2021
}

\begin{abstract}
The rapid growth of mobile data traffic has caused great pressure on the limited spectrum resources, and there must be some better methods to deal with this problem. The innovative technology of Long-Term Evolution (LTE) using the unlicensed spectrum, known as LTE-Unlicensed (LTE-U), has been proposed to effectively alleviate the shortage of authorized band resources. LTE-U has explored a lot of potential capacity in mobile communication systems with limited authorized spectrum resources, and improved the spectrum utilization of unauthorized frequency bands. However, LTE-U is still facing challenges in its application. In this paper, we summarize the key features of LTE-U and the coexistence of LTE-U with Wi-Fi in the unlicensed Spectrum. We analyze the key technologies (including carrier aggregation, HARQ, interference cancelation, and centralized scheduling), the operating modes and deployment scenarios (including carrier aggregation LTE-U, duty cycle LTE-U, and standalone LTE-U), and the advantages (including anchored LTE-U and Standalone LTE-U scenarios), as well as main technical challenges. We then address the different management mechanisms of LTE-U and Wi-Fi (including the differences between the MAC layer and physical layer), the types of coexistence technology classification (including channel separation and channel sharing technologies), and directions for future work. We hope that this comprehensive survey spurs further research in this promising area.
\end{abstract}

Keywords: LTE-U; unlicensed spectrum; coexistence technology

\section{Introduction}

With the development of the mobile Internet, the number of smart devices and mobile applications is growing rapidly. With the implementation of smart devices, people can communicate and connect anytime and anywhere. From 2017 to 2022, mobile data traffic increased by seven times, and by 2022, the monthly global mobile data traffic is projected to be $77.5 \mathrm{~EB}$ [1]. At the same time, the Internet of Things (IoT) has also ushered in the rapid development of mobile networks, which has brought explosive traffic demands [2]. The business types and business demand of mobile networks have increased unprecedentedly, which has resulted in higher requirements on communication capacity, speed, delay, and

This work is licensed under a Creative Commons Attribution 4.0 International License, which permits unrestricted use, distribution, and reproduction in any medium, provided the original work is properly cited. 
security. However, the limitation of spectrum resources remains a challenge and to be solved by new communication technologies. Additionally, users' needs are constantly escalating. From simple voice communication and web browsing in previous decades, to the current video-based social interaction, the average usage of mobile users continues to hit new highs, and the requirements for the mobile networks are increasing.

Cellular mobile communications systems adopt a cellular wireless networking mode, supports terminal movement, and can hand over automatic roaming across local networks. Thus far, the global penetration rate of cellular network has surpassed that of fixed communication networks, making them the most widely used wireless communication networks. It took 20 years for the industry to realize the development from the Second-Generation Mobile Communication Technology (2G) to the Fourth-Generation Mobile Communication Technology (4G). Long-Term Evolution (LTE) is based on Orthogonal Frequency Division Multiple Access (OFDMA) technology and Multiple Input Multiple Output (MIMO) technology, which enables the system to have four-dimensional transmission resources: the time domain, frequency domain, spatial domain and code domain. LTE has brought better quality of service (QoS) to users, making 4G communication technology the most widely used network for cellular mobile communication in the world [3]. The Fifth-Generation Mobile Telecommunications (5G) has recently become a global hot spot, and was put into commercial use in 2020. Compared with the previous generations of cellular mobile communications, $5 \mathrm{G}$ will face more diverse scenarios and differentiated performance requirements [4]. The International Mobile Telecom System (IMT) 2020 (5G) Promotion Group of China, starting from the $5 \mathrm{G}$ vision and needs, summarized the main $5 \mathrm{G}$ technical scenarios, key challenges and applicable key technologies. A Google whitepaper proposes that technical indicators of $5 \mathrm{G}$ include the connection number density, end-to-end delay, mobility, peak rate, traffic density, user experience rate, spectrum efficiency, energy efficiency, and spectrum efficiency, which could shorten the distance between everything [5].

WLAN developed rapidly in the 1990s. It uses radio frequency (RF) technology to interconnect smart devices and form a network that could communicate and share resources. WLAN has been widely used because of its simple and flexible deployment, high transmission rate, and large capacity. The most common series of standards in WLAN is IEEE 802.11, which includes IEEE 802.11 a, IEEE $802.11 \mathrm{~b}$, IEEE $802.11 \mathrm{~g}$ and IEEE $802.11 \mathrm{n}$ protocols. Currently, the 802.11 series of protocols are commonly known as wireless fidelity (Wi-Fi), which is a band based on the IEEE 802.11 series of standards, and is dedicated to solving IEEE 802.11 production and equipment compatibility issues [6].

The existing research shows that LTE-U could provide better coverage, larger capacity, higher spectrum efficiency, compared with LTE, as well as seamless data transmission between licensed and unlicensed spectrum [7]. However, LTE-U still faces two major challenges. The first challenge is the coexistence of the LTE-U system and existing systems on the unlicensed spectrum, such as Wi-Fi systems. Studies there have investigated this coexistence of LTE-U and Wi-Fi systems in unlicensed frequency bands. Dandachi et al. [8] introduced the coexistence mechanism of LTE-U and Wi-Fi systems in unlicensed frequency bands. Simulation results showed that the LTE-U system accessing the unlicensed spectrum will seriously affect the Wi-Fi system. To ensure the transmission performance of the Wi-Fi system and protect it from severe interference by LTE-U, a suitable access mechanism must be adopted to restrict the transmission of LTE-U in the unlicensed frequency band. The coexistence of LTE-U and Wi-Fi can be referred to the coexistence of secondary users and primary users as a cognitive radio technology, which is achieved by underlay, interweave and overlay modes. In the overlap mode, if the interference of the secondary user to the primary user is lower than the interference threshold, the secondary user and the primary user are allowed to transmit information at the same time. In the coverage mode, different signal processing and coding techniques are used to distinguish the signals between the primary user and the secondary user. When the primary user occupies the channel, the secondary user could access the channel without 
causing interference. Therefore, the LTE system could use overlap and coverage modes for transmission [9], and simultaneously use the unlicensed spectrum with the Wi-Fi system. Choi et al. [10] proposed a duty cycle (DC) mechanism, that is, LTE-U occupies a certain proportion of the transmission time in each frame, and then LTE-U stops transmission so that the Wi-Fi system has a chance to access it, thus ensuring fairness in the transmission ratio between LTE-U and Wi-Fi systems. Zhao et al. [11] proposed a dynamic spectrum access scheme based on channel detection results to realize sharing of time domain spectrum resources. Heng et al. [12] proposed an enhanced frame-based equipment (E-FBE) mechanism based on Listen Before Talk (LBT). This solution is similar to the access method of Wi-Fi contention fallback, thereby avoiding conflicts between LTE-U users. Nevertheless, this solution will cause a long time fallback and reduce spectrum utilization. Liao proposed a discrete spectrum access mechanism based on full-duplex technology, and capable of simultaneous data transmission and channel state detection [13]. In this solution, the mobile terminal of each user is equipped with two full-duplex antennas, Ant1 and Ant2. Ant1 continuously detects the channel state. When the channel is detected as idle, Ant2 sends data. Lien et al. [14] proposed an optimal decision mechanism for random or scheduled access to unlicensed frequency bands.

The rest of the present paper is organized as follows. As shown in Fig. 1, Section 2 reviews current status about the LTE-U. Section 3 describes LTE-U and Wi-Fi coexistence on an unlicensed spectrum. Finally, Section 4 concludes the paper.

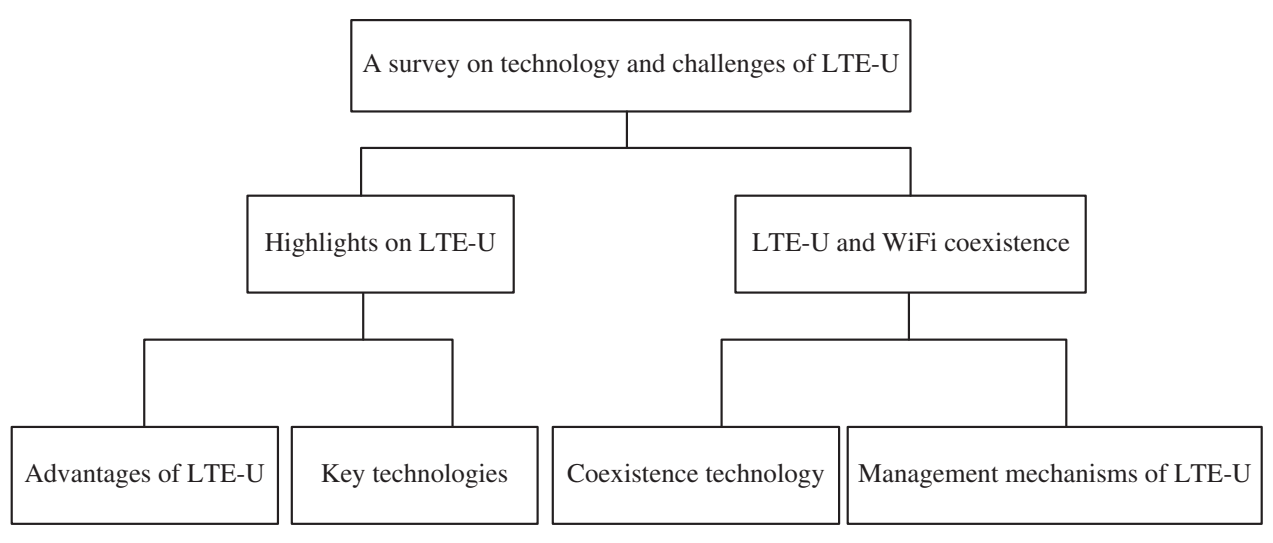

Figure 1: Structure of this paper

\section{Highlights on LTE-U}

This section will introduce several highlights of the LTE-U system, including the choice of unlicensed spectrum, the key technologies in LTE-U, the operating mode and deployment scenarios of LTE-U, and the advantages and research hotspots of LTE-U.

\subsection{Unlicensed Spectrum}

Unlicensed frequency bands refer to spectrum resources that could be used in accordance with regulations without the permission of the administrative department. The Federal Communications Commission (FCC) has released multiple frequency bands for unlicensed commercial usage, including the $2.4 \mathrm{GHz}$ industrial, Industrial Scientific Medical (ISM) band, the $5 \mathrm{GHz}$ Unlicensed National Information Infrastructure (U-NII) band, and $60 \mathrm{GHz}$ millimeter wave frequency band. In recent years, the FCC has allocated some new frequency bands for market selection, such as, the $6 \mathrm{GHz}$ frequency band for Wi-Fi $6 \mathrm{E}$ and $5 \mathrm{G}$, and multiple frequency bands on high-band frequency. Among them, $2.4 \mathrm{GHz}$ is the most commonly used yet crowded frequency band on which already runs WLAN, ZigBee, Bluetooth and other systems. The $5 \mathrm{GHz}$ band is mainly used for Wi-Fi networks. Compared with 
$2.4 \mathrm{GHz}$, it has more available channels, and is less crowded, but has larger signal attenuation. Furthermore, its wall penetration ability is relatively weaker and the propagation distance is shorter. The $60 \mathrm{GHz}$ frequency band is free, with $6000 \mathrm{MHz}$ bandwidth available, but the electromagnetic wave can be absorbed by oxygen. Thus, a more complex physical layer and air interface design are required [15]. However, most of the current deployment studies for LTE-U are based on the $5 \mathrm{GHz}$ frequency band, which is excellent in all aspects.

Fig. 2 presents the spectrum distribution around $5 \mathrm{GHz}$ in some countries and regions. Among them, the US 5.15-5.35 GHz, 5.47-5.85 GHz, a total of $550 \mathrm{MHz}$ is open as an unlicensed frequency band. In Europe, 5.15-5.35 GHz and 5.47-5.725 GHz are only available for wireless access systems (WAS), and the European Commission is proposing to open the 5.725 v $5.85 \mathrm{GHz}$ frequency band, which is currently used as an unlicensed frequency band for fixed wireless access (FWA) and intelligent transportation systems (ITS). In China, the 5.15-5.35 GHz unlicensed frequency band is only used for indoor wireless communication, and the 5.725-5.85 GHz unlicensed frequency band can be used both for indoor and outdoor wireless communication [16]. In summary, the available resources of the $5 \mathrm{GHz}$ frequency band are rich in various regions, and can accommodate tens of $20 \mathrm{MHz}$ channels.

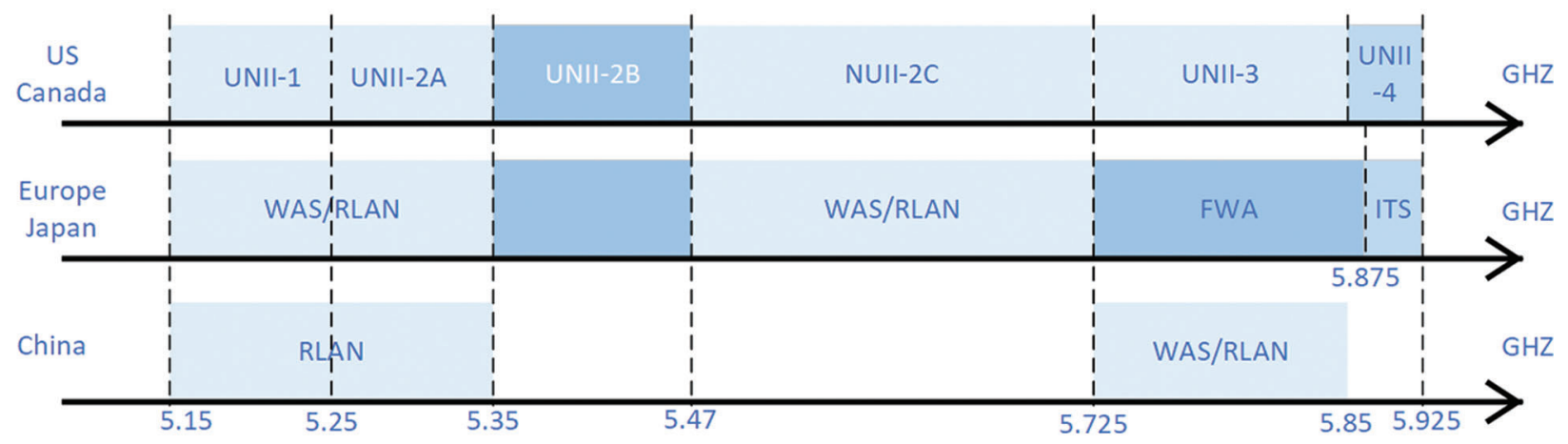

Figure 2: $5 \mathrm{GHz}$ band spectrum layout in different regions

\subsection{Key Technologies of LTE-U Systems}

The advantage of the LTE-U system lies in that it makes full use of the existing architecture and technology of LTE. Compared with Wi-Fi, LTE has superior system performance and long-term development technical background. LTE-U has higher spectrum utilization efficiency and more stable performance in the entire system than Wi-Fi. In the following, we describe the key technologies needed for the implement the LTE-U system: carrier aggregation (CA), hybrid automatic retransmission (HARQ), interference control (IC), and centralized scheduling.

\section{(1) Carrier Aggregation}

Carrier aggregation (CA) is the aggregation of two or more component carriers (CCs) to support a more extensive transmission bandwidth (maximum to $100 \mathrm{MHz}$ ). 3GPP was introduced CA in Release 10 as part of LTE-A, and further expanded in Release 11. CA is a key technology in LTE-U, making the unlicensed spectrum controllable. Since the spectrum resources of the unlicensed frequency bands are scattered relative to the licensed frequency bands and have different physical properties, it is difficult to run normally in both spectrums. However, CA can use multiple different frequency bands for unified transmission simultaneously. Generally, the carrier of the licensed spectrum is the primary carrier in LTE-U, and multiple carriers of the unlicensed spectrum are the subcarriers; then, LTE-U uses the primary carrier and subcarrier to aggregate carriers. 


\section{(2) HARQ}

HARQ is a technology used between the receiver and the sender in the LTE network. When a receiver receives a data packet from the sender, the receiver will not directly discard the information if the received data information is wrong. The receiver chooses not to send the ACK feedback signal, and waits for the sender to re-send the data information. Then the receiver integrates the data information according to certain specifications. In the Wi-Fi system, system users need to re-listen to channel competition for access once data transmission fails or collision occurs. In contrast, LTE-U technology can use system resources to improve utilization and increase the success rate of transmission.

(3) Interference Cancelation

The structure of LTE-U is similar to that of LTE, so the inter-cell interference will be encountered. Generally, interference control methods in the cellular network are as follows: interference cancelation (IC), inter-cell interference cancelation (ICIC), and interference randomization (including scrambling, interleaving division multiple address, and frequency hopping).

IC demodulates and decodes the signals of between a cell and neighboring cells with the same frequency, and uses the correlation to separate the respective interference signals and the useful signals.

ICIC selects different time-frequency resources by coordinating between a cell and its neighboring cells. The ICIC module is used to determine which time-frequency resources or transmission power of a specific time-frequency resource can be used by the resource scheduler, thereby avoiding the inter-cell interference.

Interference randomization uses the mature scrambling technology of the CDMA system, which is relatively simple and feasible. However, the interference is treated as white noise, which may cause measurement errors due to different statistical characteristics. An unlicensed spectrum inter-cell interference coordination (usICIC) mechanism to improve system performance was proposed in [17]. In usICIC, a coordination framework for dynamic spectrum management is established between multioperator and multi-technology networks [18]. The existing literature mainly studies how to use cooperation mechanisms to ensure fair coexistence, reduce interference, and allocate resources. However, cooperative communication also improves data rate and spectrum efficiency through joint transmission and cooperative reuse.

\section{(4) Centralized Scheduling}

Centralized scheduling means that all system resources, including time, frequency, and space resources are uniformly divided and configured by the macro base station. The user terminal does not need to participate in resource competition.

The application of this technology with the LTE-U system can effectively prevent collisions between the data services of the LTE system and the data services of the Wi-Fi system, thereby rationally and effectively utilize the system's spectrum resources, and reducing spectrum waste. However, this technology also has its limitations. With the explosive growth of network equipment in the Internet of Things era, it is impossible to complete such a huge resource scheduling by a base station alone. Generally, for scenarios with dense terminal equipment, it is necessary to deploy multiple large base stations with centralized scheduling functions to meet the necessary QoS.

\subsection{Operating Modes and Deployment Scenarios}

From Release 13 to Release 17, LTE-U technology has gradually matured, and multiple development directions and implementation methods have been formed. The main operating modes and deployment scenarios of LTE-U are discussed in the following subsections. 


\subsubsection{Modes of Operation}

As show in Fig. 3, three operating modes are mainly considered in LTE-U [19].

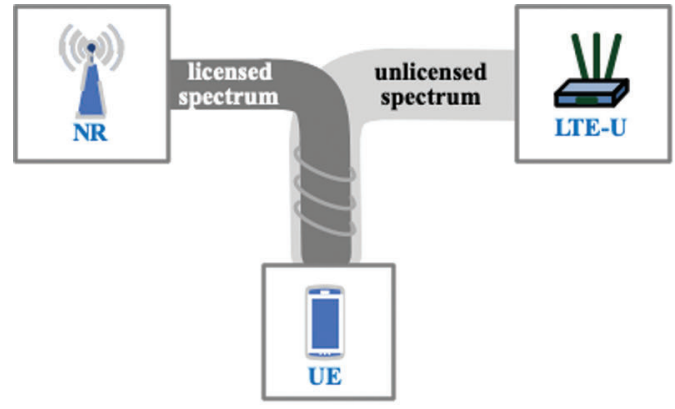

Carrier Aggregation

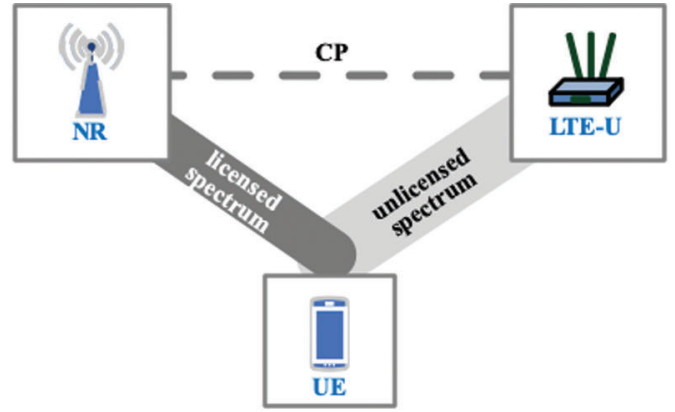

Dual Connection

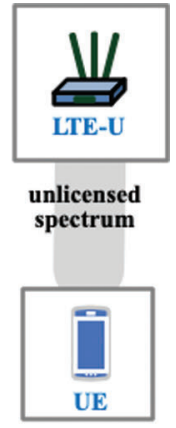

Standalone

Figure 3: Three operating modes of LTE-U

(1) CA LTE-U is based on LTE-LAA, and introduced in Release 13. CA uses an aggregate of LTE-U in the unlicensed band and LTE (or NR) in the licensed band. This mode can be subdivided into supplementary downlink (SDL) and supplementary uplink (SUL) modes for different usage scenarios.

CA uses the licensed spectrum of the proper frequency band as the main carrier PCC, and several carriers of the unlicensed spectrum as the subcarrier SCC, and then aggregates multiple carriers for transmission to serve the same user. It should be noted that the control signal and the data signal can be simultaneously transmitted on the PCC. In contrast, only the data signal is transmitted on the SCC without the control signal. This is because the LTE system in the licensed frequency band has less interference yet more stable performance, and can better guarantee the user's quality of service (QoS). Meanwhile, the LTE system could well regulate the unlicensed carrierby adding or subtracting subcarriers to usage requirements.

In most scenarios, the downlink network traffic will far exceed the uplink network traffic. Only the use of the unlicensed spectrum for downlink transmission would relieve the downward pressure on the LTE system. Therefore, the LTE-U system only needs to perform data downlink transmission on the unlicensed spectrum while simultaneously performing uplink and downlink transmission on the licensed spectrum. This deployment scheme is called SDL [20]. It could be found in 3GPP Release 15 that 3GPP introduced SUL in $5 \mathrm{G} \mathrm{NR}$ to solve the higher requirements of various services forms on network uplink capabilities. Like to SDL, SUL uses unlicensed frequency bands as a supplement to the uplink transmission link, thereby improving the entire system's uplink capacity.

(2) Dual connection (DC) LTE-Uwas proposed by 3GPP in Release 12 and is used to enhance the capabilities between cells. In this mode, the terminal can configure multiple reception and transmission (RX/TX) systems in the RRC, and apply two different schedulers to schedule radio resources. The two schedulers are located in two eNodeBs, namely, the saster eNodeB, and the secondary eNodeB. They are connected in a non-ideal backhaul way through the X2 interface. The difference between DC and CA is that CA usually uses multiple carriers of a different frequency band in one single cell for aggregate transmission, while DC uses the same frequency band in different cells for transmission. CA technology is also used in DC, that is, for two different cells, CA is performed in each cell, and then the scheduler transmit $\mathrm{CA}$ and $\mathrm{DC}$ together through dual connection.

(3) Standalone (SA) LTE-U is a novel approach in 3GPP Release 16. When SA LTE-U works in the unlicensed spectrum without being anchored to any licensed operators, CA and DC modes are collectively referred to as anchored LTE-U because they use the licensed spectrum as an "anchor". This is 
similar to the solution MulteFire proposed for LTE. The SA mode in the MulteFire solution is different from the anchored LTE-U because it does not need to set an "anchor" in the licensed spectrum, and can operate independently in the unlicensed spectrum. The LTE-U system in this mode is more flexible and versatile than CA and DC, and is especially suitable for areas without LTE coverage; moreover, it can be deployed in any scenario, like alongside a Wi-Fi system. However, it is not supported by a licensed spectrum, and its control signal runs in an unlicensed spectrum; thus, MulteFire's reliability of communication transmission and the effectiveness of resource management are not as good as those of anchored LTE-U, but it is usually regarded as a good substitute for Wi-Fi. The MulteFire registered by Qualcomm is a good representative of the SA mode. Studies have shown that when MulteFire and Wi-Fi are deployed together, and when the neighbor switches to MulteFire, Wi-Fi performance is preserved and sometimes improved [21].

\subsubsection{Deployment Scenarios}

With the standardization of $5 \mathrm{G}$ New Radio (NR), 3GPP has conducted research on NR Unlicensed (NR-U) in Release 16. Five deployment scenarios for NR-U have been identified in the 3GPP report [22]:

Scenario A: CA between NR-U and licensed frequency band NR.

Scenario B: Dual connection between licensed band LTE and NR-U.

Scenario C: Standalone NR-U.

Scenario D: NR unit for downlink in the unlicensed frequency band and uplink in the licensed frequency band.

Scenario E: Dual connection of NR-U and licensed frequency band NR.

Note that scenario, scenario A is similar to License Assisted Access (LAA), and scenario C is similar to MulteFire [23]. The five deployment scenarios of NR-U (LTE-U) are also shown in Fig. 4.

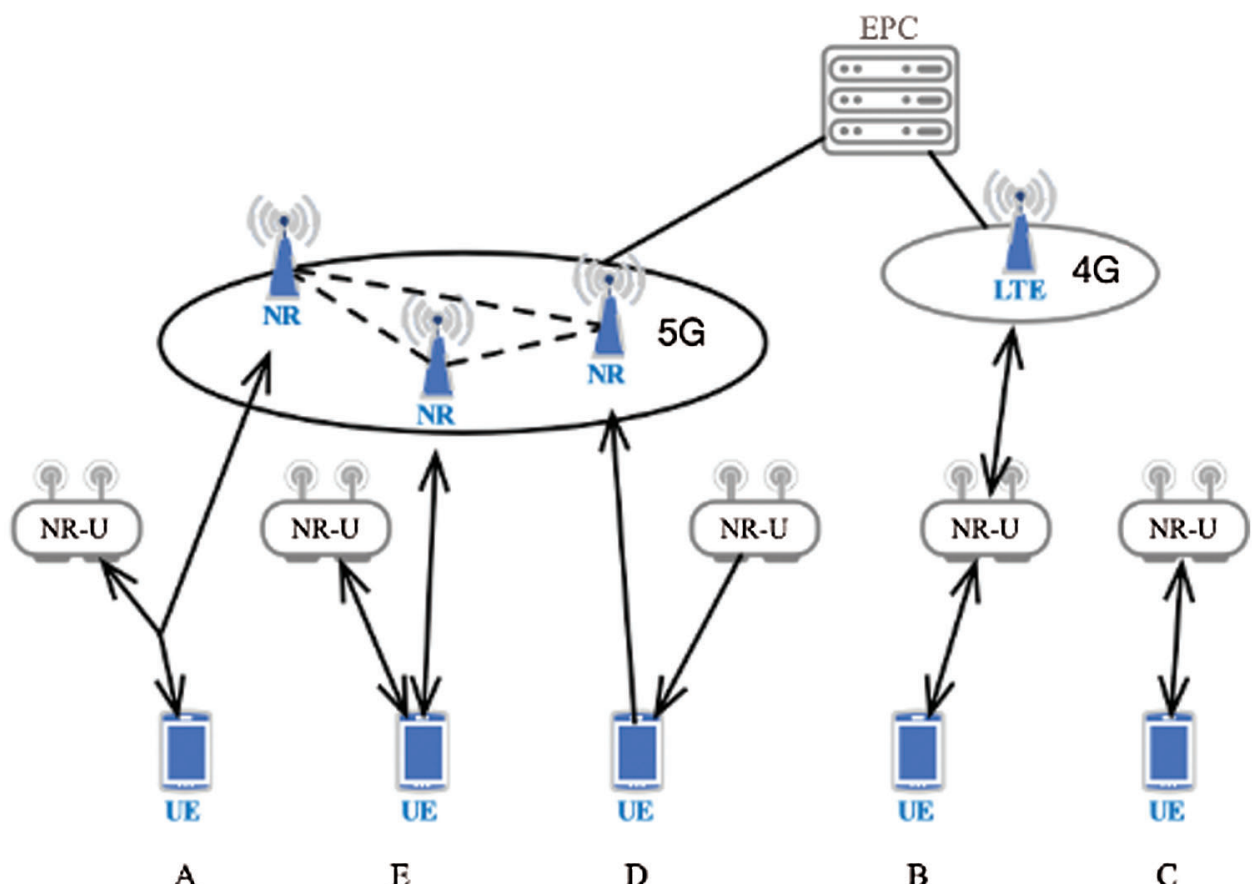

Figure 4: Deployment scenarios of NR-U(LTE-U) U). Five deployment scenarios are composed of the followings: (A) (CA between NR-U and licensed frequency band NR), (B) (dual connection between licensed band LTE and NR-U), (C) (standalone NR-U), (D) (NR unit for downlink in the unlicensed frequency band and uplink in the licensed frequency band), (E) (dual connection of NR-U and licensed frequency band NR). 


\subsection{Advantages of LTE-U}

The CA and DC modes use both the licensed and unlicensed spectrum in the LTE-U working mode while the SA mode only applies to the unlicensed spectrum; thus, these modes have different application scenarios and advantages.

\subsubsection{Advantages of Anchored LTE-U}

Anchored LTE-U has the following advantages:

(1) Improving the data transmission rate on $5 \mathrm{GHz}$ band

First of all, LTE-U uses both licensed and unlicensed spectrum and uses CA technology to expand the bandwidth several times, thereby achieving faster transmission speed and greater throughput. In addition, the Wi-Fi system uses random access based on constraints and can only communicate with one user at a time. In a user-intensive and traffic-intensive indoor environment, the user experience is often unsatisfactory. However, LTE uses scheduling-based channel access and scheduling data transmission pipes based on channel quality to achieve multi-user frequency selection diversity gain. The LTE system employs many advanced technologies for resource allocation and user scheduling, such as elCIC, and the coordinated multipoint transmission mechanism CoMP.

(2) Providing better continuity and mobility

When a Wi-Fi user leaves the coverage area of a Wi-Fi node, the UE must disconnect the current connection and re-establish a connection with LTE to continue communication. This kind of handover between different communication systems will cause discontinuity of UE communication, and the UE usually senses service interruption. At the same time, the security risk of the UE is increased, and more overhead is required to perform security checks. The control information of the UE is transmitted on the PCC of the licensed spectrum, and the PCC of the licensed spectrum provided by the LTE system is ubiquitous. Therefore, the handover only needs to offload the data transmission on the unlicensed spectrum to the licensed spectrum, and the user side hardly feels service interruption. At the same time, the LTE-U system inherits the mobility of the LTE system, which is impossible in a Wi-Fi system. In fact, some researchers have used LTE-U in high-speed mobile communications.

(3) Reducing the cost of operators

Since the LTE-U system is built on LTE, the initial cost of building the LTE-U system is significantly reduced. At the same time, operators no longer need to spend a lot cost on purchasing a new licensed spectrum. The use of an unlicensed spectrum can greatly increase the rate of the communication system, reduce the average cost, and benefit operators.

\subsubsection{Advantages of Standalone LTE-U}

The SA LTE-U structure is different from that of the anchored LTE-U since it allows transmission on the unlicensed spectrum without an anchor in the licensed spectrum. Thus, SA LTE-U has unique characteristics. Here, we take MulteFire as an example. It has the following advantages [24].

(1) Plug-and play deployments: Since there is no need for carrier aggregation with the carrier of the licensed frequency band, and no unified scheduling with LTE, the equipment structure is simple, the deployment cost is low, and plug-and-play is possible. Hence, compared with Anchored LTE-U, the flexibility is higher.

(2) Expands IoT opportunities: MulteFire is very suitable for deployment in resource-intensive environments, such as the Internet of Things. Moreover, it has better performance than Wi-Fi in crowded communication conditions. 
(3) Better security: MulteFire serves any users with LTE-like security, which has excellent security. In contrast, Wi-Fi usually only provides deficient security.

(4) Expand coverage: MulteFire expands mobile network data and services indoors, while achieving faster resource unloading than Wi-Fi; it also improves the performance of the entire communication system.

\subsection{Main Technical Challenges of LTE-U}

LTE-U runs on the unlicensed spectrum, which has been used by other communication devices. Many Wi-Fi devices already exist in the most applicable $5 \mathrm{GHz}$ frequency band. A Qualcomm whitepaper shows that LTE-U is a friendly neighbor of Wi-Fi [25], but t Google's whitepaper points out that the deployment of LTE-U will seriously deteriorate Wi-Fi network performance. We can thus see that there is a problem of coexistence on the unlicensed spectrum of LTE-U and Wi-Fi.

From the perspective of communication and transmission, LTE does not require monitoring to access transmission while Wi-Fi networks use a distributed control function (DCF)-based MAC layer protocol (DCF is a multi-access/conflict avoidance based on carrier sensing). The transmission mechanism of distributed access technology mainly uses requests to send/clear (RTS/CTS); that is, an RTS signal will be sent before the data is transmitted. When the UE receives the RTS signal, it will send a CTS signal to the sending station. Only after the sending station receives the CTS signal will it begin to transmit data, and it continues to do so until the ACK signal appears. When LTE and Wi-Fi coexist, the RTS sent by the Wi-Fi sender will be interfered by the continuous control data of LTE, and cannot be accurately received by the UE because the control data of the LTE system is continuously sent. Therefore, Wi-Fi will always be in a waiting state, and sending RTS signals repeatedly until LTE-U becomes idle. LTE-U still periodically sends control signals. When LTE is not transmitting data, it still sends control signals, which cause the Wi-Fi to have almost no time to transmit data [26]. Therefore, it is necessary to propose a technical solution that allows LTE-U and Wi-Fi to coexist friendly.

In short, the purpose of spectrum resource reallocation is to achieve higher spectrum utilization while ensuring that the quality of service of Wi-Fi and LTE-U is not affected.

\section{Coexistence of LTE-U with Wi-Fi in Unlicensed Spectrum}

Since LTE-U and Wi-Fi have been developed independently for many years, there are many different access methods. To realize the harmonious coexistence of LTE-U and Wi-Fi system, the primary problem to be solved is how to reduce the interference of Wi-Fi due to the unauthorized use of LTE-U frequency band.

\subsection{Different Management Mechanisms of LTE-U and Wi-Fi}

When using LTE-U in the unauthorized frequency band, the first problem to be solved is its friendly coexistence with the Wi-Fi system [27]. Although LTE-U would be a friendly neighbor to Wi-Fi, the deployment of LTE-U would seriously deteriorate the performance of Wi-Fi [28]. Because LTE-U and Wi-Fi use different radio access technologies (RAT) and have developed independently for many years, they have great differences in the medium access control (MAC) layer and the physical layer (PHY).

(1) MAC layer

The LTE-U system uses the same central control protocol in the MAC layer as the LTE system, and it has a continuous transmission mechanism. Wi-Fi adopts the protocol of the MAC layer based on the distributed coordination function (DCF). The DCF is a distributed access mechanism based on carrier sense multiple access with collision avoidance (CSMA/CA), which means that Wi-Fi does not have a central control. The CSMA/CA mechanism detects whether the channel is idle before each Wi-Fi user accesses the 
channel to transmit data. The CSMA/CA mechanism will listen for the distributed interframe spacing (DIES) time, and datee are sent if the channel has been idle for the duration of the listening time.

A fallback count is randomly selected within the competition window until its value decreases to 0 . Then the channel is re-detected until the channel is idle and data transmission begins, which reduces the probability of collisions between Wi-Fi users. When many wireless users are competing for the frequency band, the frequency spectrum utilization will be low because of the conflict avoidance mechanism of Wi-Fi [29].

(2) Physical layer

LTE and Wi-Fi networks are entirely different in physical layer characteristics [30]. The physical layer of the LTE-U system adopts OFDMA technology. Its bandwidth is up to $100 \mathrm{MHz}$ and can be divided into a series of physical resource blocks (PRBs). Each PRB is composed of 12 OFDMA carriers. LTE can maximize the network performance by assigning different PRBs on the same subframe for different users. Wi-Fi uses the orthogonal frequency division multiplexing (OFDM) technology in the physical layer, which only allows a single user to use one channel at a time. In addition, the symbol cycles of these two systems are also different. The symbol cycle of LTE is 71.4, while Wi-Fi is 4 [30]. As a result, LTE and Wi-Fi cannot occupy the same channel simultaneously.

In addition to the performance differences between the physical layer and the MAC layer, Wi-Fi is widely used for enterprises and private users. Since LTE-U is mainly accessed through the low-power wireless access small cell (SC), and is usually deployed in indoor locations, operators may face the complex decision of choosing between LTE and Wi-Fi [31].

To sum up, the key to the implementation of LTE-U technology is to solve the coexistence problem of LTE and Wi-Fi networks in the unlicensed frequency band. For this purpose, it is necessary to design a new scheme for resource allocation of the LTE-U system and to explore a new LTE-U protocol that can coexist fairly with the Wi-Fi system.

\subsection{Coexistence Technology Classification}

Existing coexistence technology is divided into two categories: channel separation technology and channel sharing technology. Channel separation technology refers to the fact that Wi-Fi service is relatively idle; The unauthorized frequency band is rich in resources and the base station can choose the idle channel for transmission. Channel sharing technology means that LTE-U users and Wi-Fi users need to share the channel and unauthorized channels need to be reused when Wi-Fi service is busy.

\subsubsection{Channel Separation Technology}

When the unlicensed frequency band resource data are much larger than that required by the Wi-Fi system, the appropriate unlicensed channel can be selected through channel selection (CHS) to transmit data as LTE-U. CHS technology has two implementation schemes: the randomly selected channel scheme and dynamically selected channel scheme.

(1) Random selection of channel scheme

The random selection channel scheme is a scheme in which small base stations randomly select unauthorized frequency band access [32,33]; the system provides multiple free indoor and outdoor channels [34]. However, due to the open nature of the unauthorized frequency band, it is difficult to ensure that a certain channel is a long-term fixed "clean" channel. Meanwhile, Wi-Fi systems can only access "clean" channels, which can seriously affect Wi-Fi user experience. Therefore, random channel selection is rarely used in coexistence schemes. 
(2) Dynamic selection of channel scheme

In the dynamic selection scheme, the base station chooses the channel according to the power and use of the channel, which solves the interference problem well. Under this scheme, the base station can select the "cleanest" channel, i.e., the one with the least interference, for transmission according to its power and use of [35]. A simple dynamic channel selection scheme is shown in Fig. 5. As the interference on the unauthorized channel increases with the access of Wi-Fi AP, the interference threshold is set on the channel. LTE-U periodically detects the interference value of the access channel and determines whether the interference threshold is exceeded. If the interference threshold is exceeded, the LTE-U base station will re-select the unauthorized channel with less interference. In addition, the setting of the interference value is based on the feedback of users, such as the channel quality indicator (CQI). This can avoid hiding terminal problems and achieve high detection accuracy. Villardi et al. proposed the dynamic selection of channels through channel perception. If the target channel is perceived as a free channel, it is considered to be an accessible channel; otherwise, dynamic selection will give up access and continue to look for an accessible channel. In the case of a small number of Wi-Fi users, some unauthorized frequency bands are not fully used. Therefore, the dynamic channel selection scheme is suitable for scenarios with a low density of Wi-Fi access points.

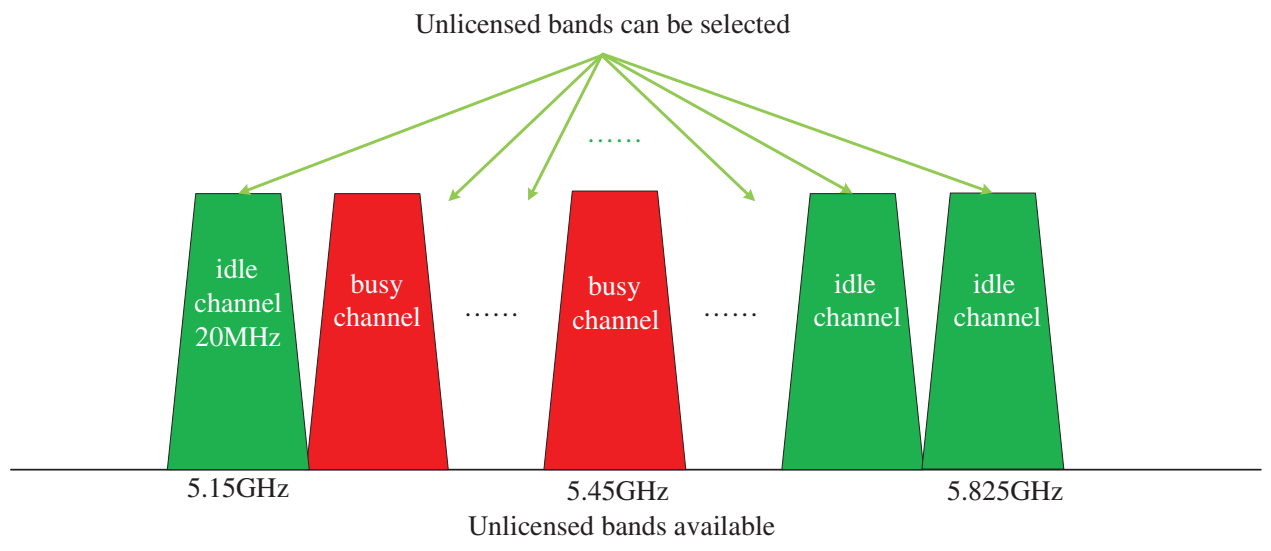

Figure 5: Dynamic channel selection mode

\subsubsection{Channel Sharing Technology}

Channel sharing technology can be divided into time domain coexistence technology and frequency domain coexistence technology. The most common methods in time-domain coexistence are the duty ratio technology and Listen Before Talk (LBT) technology; meanwhile, dynamic frequency selection (DFS) technology is the most common method in frequency domain coexistence technology [36].

(1) Time domain coexistence

For high-density deployment scenarios of Wi-Fi access point (AP), such as inside malls, airports, and companies, the coexistence of LTE-U and Wi-Fi in the time domain has to be considered. It can be seen from Fig. 6 that different countries or regions have different provisions on the use of unlicensed frequency bands. The channel sharing coexistence mechanism is divided into areas requiring an LBT access mechanism and areas not requiring an LBT access mechanism. The coexistence mode based on LBT was adopted in the areas with a specified LBT access mechanism, and the coexistence mechanism based on duty cycle was adopted in the areas without a specified LBT access mechanism. 


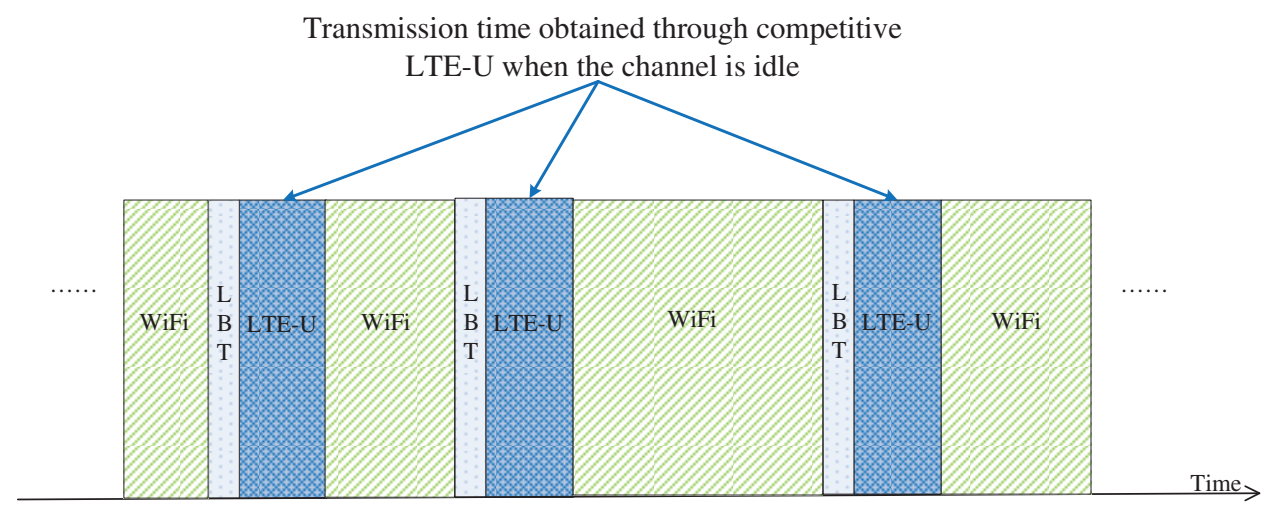

Figure 6: Coexistence way of LTE-U and Wi-Fi based on LBT

\section{A. Coexistence mode based on LBT}

Europe, Japan, and other countries that allow LBT access adopt LBT coexistence. Before data transmission, the user or small base station in the LTE-U system will listen to the channel and judge the state of the target channel. If the target channel is idle, the user or small base station can access the channel. Otherwise, they will back off for some time, as shown in Fig. 6. "Wi-Fi" means the unauthorized frequency band occupied by Wi-Fi, and "LTE-U" means the unauthorized frequency band occupied by the LTE-U system. Currently, there are three realization main LBT modes: no retreat mode, collision and retreat mode, and carrier sensing (CS) value mode.

(i) No retreat mode: The LTE-U base station or the user detects the channel before each cycle begins. If the channel is not idle, access will be given up; otherwise, the channel will be accessed [37].

(ii) Collision and retreat mode: This mode is similar to Wi-Fi DCF protocol, which was implemented in Release 13. When LTE-U stations compete with Wi-Fi users with the same unauthorized channel, the LTE-U base station will retreat if the channel collision rate is higher than Wi-Fi users or occupied [38]. Song studied the optimal value of fixed retreat window length aiming at maximizing system throughput [39]. Some researchers have discussed the coexistence performance of LTE and Wi-Fi, when the length of the retreat window is dynamically variable [40-42]. Meanwhile, Lee proposed a fair LBT algorithm based on the dynamic selection of the contention window (CW) length scheme [39]. This algorithm not only guarantees the overall performance of the system, but also provides fairness for LTE and Wi-Fi systems. Yin suggested that by modifying the length of $\mathrm{CW}$, the system under the LBT protocol could have a higher access probability and effectively improve the performance of the system [41]. Tao proposed a scheme to ensure the fairness of quality-of-service (QoS) by adjusting the length of CW [42]. The advantage of this coexistence method is to reduce the mutual interference when LTE-U and Wi-Fi coexist.

(iii) Carrier sensing (CS) value mode: This mode was proposed by the European telecommunications Standards Institute (ETSI) to control the access mode of the LTE-U system [43]. The main technique in this method is to take the signal capability level (interference level) on the target channel as a parameter. If the energy level is lower than the preset threshold, the LTE-U base station can access the channel. Otherwise, it will continue to wait for an accessible channel. The preset threshold is based on the decision of jammer Equivalent Isotropically Radiated Power (Equivalent Isotropically Radiated Power, EIRP). Han discussed the impact of fixed CS threshold on system performance and researched how to improve the overall performance of the system by modifying the MAC layer protocol [44]. Li et al. [45] studied the dynamic adjustment of the CS threshold to achieve spectrum reuse, and improve spectrum utilization. 


\section{B. Coexistence mode based on duty-cycle mechanism (DCM)}

A DCM was proposed by academia and industry in non-LBT regions, such as in the United States, South Korea, and China. DCM is the LTE-U system that divides the unauthorized frequency band into fixed periods in the time domain, by alternating $\mathrm{ON}(\mathrm{ON}) / \mathrm{OFF}$ (duty cycle) modes during each cycle. The ON time of a cycle is used for LTE-U transmission, and the OFF time is used by Wi-Fi. The accessing systems are separated in time so as not to interfere with each other. However, in the case of a fixed duty cycle, the required transmission time of LTE-U and Wi-Fi cannot be determined, and this will affect the improvement of spectrum utilization of the unauthorized frequency band [46]. Therefore, the duty cycle needs to be dynamically adjusted. Qualcomm proposed a duty cycle mode based on carrier sensing adaptive transmission (CSAT) [25]. CSAT technology is used to dynamically turn on or off the LTE-U base stations deployed in the unlicensed frequency band by periodically detecting channel conditions. A CSAT cycle lasts about 20-100 ms. The difference between CSAT and DCM is that when the base station shuts down its data transmission module to give access to the channel, the state of the channel will still be detected. The LTE system can carry out an adaptive scheduling according to the Ei-Fi usage (duration, etc.) of the channel in the next cycle. As shown in Fig. 7, this method utilizes the target channel through CS technology, and adjusts the switching cycle and duty ratio of LTE-U and Wi-Fi adaptably. CSAT works well with LTE standards. To ensure the detection accuracy, sufficient listening time is needed. Furthermore, the CSAT switching cycle is usually set to several hundred milliseconds. This results in a severe delay for LTE-U users. Researchers have discussed the effects of different duty cycles and switching cycles on LTE-U and Wi-Fi coexistence systems [47-49]. Rupasinghe proposed a scheme using a $\mathrm{Q}$ learning algorithm to find the best duty cycle in $20 \mathrm{~ms}$ [50].

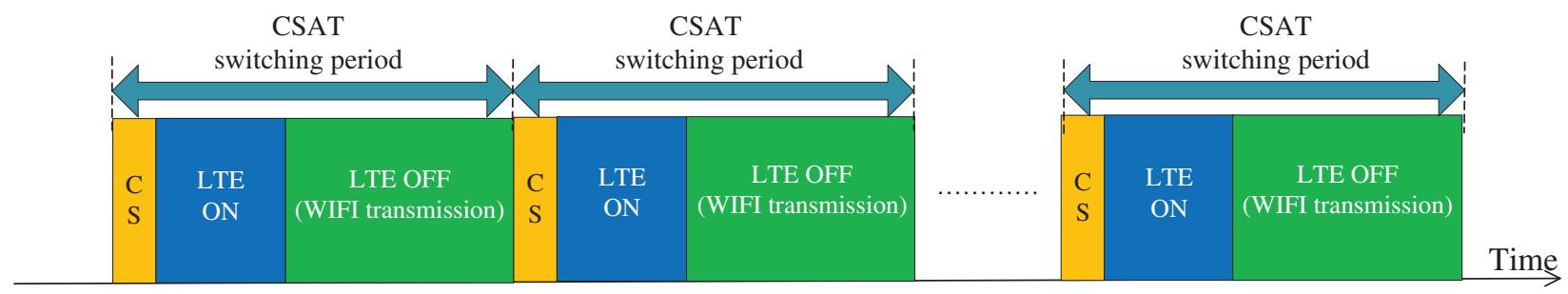

Figure 7: Coexistence of LTE-U and Wi-Fi based on CSAT

In the coexistence mode of duty cycle, together with the two modes mentioned above, there are three improved coexistence modes.

(i) Silent LTE sub-frame mode: due to the strong flexibility of LTE system, the proportion of sub-frames can be configured according to the business requirements, if the traffic volume of upstream and downstream channel transmission of LTE is quite different. Thus, the coexistence mode of LTE muting was proposed, that is, LTE-U does not transmit data in some subframes, and Wi-Fi can use the resources of these subframes [51].

(ii) Almost-blank subframe (ABS): This technology transmits the free time slot to Wi-Fi users to achieve the duty ratio function. Zheng et al. [52] improved ABS technology by preventing LTE-U from occupying a channel for too long, thus ensuring the harmonious coexistence of the two systems.

(iii) Blank subframe (BS): Compared with ABS, BS had improved by enhancing the coordination of inter-layer interference and setting almost-Blank Subframe to absolute "Blank" [53]. This scheme retains the high synchronization of LTE, and can improve spectrum utilization. However, there is still room for improvement. The number of BSs can be dynamically adjusted according to the transmission demand of Wi-Fi. Finally, in order to achieve a fair coexistence of LTE-U and Wi-Fi, it is particularly important to restrict number and coordinate BS. 
There are still many problems that require further study. First, in both LBT and the duty cycle mode, access of the permission-free frequency band is actively controlled by the LTE-U system and the performance of the Wi-Fi system is not well considered. How to adaptively adjust the access parameters of LBT or the duty cycle mode according to the network status, channel environment and service requirements of the LTE-U and Wi-Fi systems to maximize the spectrum efficiency of the unlicensed frequency band must be further studied. Additionally, more attention must be paid to how to realize the fairness between these two systems. Secondly, LBT and the duty cycle lack central control, which exacerbates the coexistence problem. When there are multiple LTE-U networks, the LBT mode adopts competitive access, and the performance decreases significantly with the increasing number of competing networks. In addition, due to the lack of a central coordination mechanism, the duty cycle mode cannot avoid the interference between different LTE-U networks. Therefore, how to make use of different system features and how to design a more harmonious coexistence scheme is an important research point.

(2) Frequency domain sharing

Many 20-MHZ bandwidth channels are now being converted to $5 \mathrm{GHZ}$. To ensure the access of different communication systems in the $5 \mathrm{GHz}$ unlicensed frequency band, some countries or regions have listed the DFS as the capability that the access network must have. DFS is the most representative technique in frequency domain sharing strategy. In the coexistence system based on DFS, the LTE-U based small cellular base station needs to have spectrum sensing technology; furthermore, it must dynamically select idle channels by sensing multiple channels. DFS plays a great role in improving the spectrum utilization of the unlicensed frequency band. It can effectively avoid the interference between LTE-U cells and other cells, and between LTE-U and Wi-Fi devices. DFS has a significant effect on medium and low density deployment scenarios, but has difficulty playing its role under a high load. Meanwhile, in consideration that 802.11AC will support a maximum bandwidth of $160 \mathrm{MHz}$, it will be very difficult to find a completely idle channel if there is a large number of deployments [54].

\subsection{Future Work}

The extension of LTE technology to the unlicensed frequency band can not only solve the current shortage of authorized spectrum resources, but also greatly improve the utilization rate of the unlicensed spectrum. It can expand the capacity of the next generation mobile communication system, which will contribute to the development of 5G technology. The area that LTE and Wi-Fi networks coexist in a friendly way on the unlicensed frequency band has become one research hotspot.

This paper introduced several typical channel access mechanisms for the coexistence of LTE-U and Wi-Fi in the unauthorized frequency band, such as the listen before talk (LBT) mechanism, carrier sensing adaptive transmission (CSAT) mechanism, and almost blank subframe (ABS) mechanism. However, only the design and application of these mechanisms have been studied, and the literature lacks theoretical modeling and analysis of these mechanisms. At present, some works have been done on the modeling and analysis of the channel access mechanism of unlicensed $d$ band LTE-U and Wi-Fi coexisting network. Although some researchers constructed Markova chain models of the LBT mechanism in the LTE-U network and the DCF mechanism in the Wi-Fi network, research in this field is still very limited, and many problems require further study [55].

\section{Conclusion}

LTE-U is an innovative technology that improves the spectrum utilization of unauthorized frequency bands. It can provides better coverage, greater capacity, greater spectrum efficiency, compared with LTE and allows seamless data transmission between the authorized and unlicensed spectrum. In this paper, we provide a comprehensive survey of LTE-U systems, and we summarized the key technologies, working 
modes, deployment scenarios, and advantages of LTE-U. We also discussed the main challenges faced by LTE-U. Several major technologies for the coexistence of LTE-U and Wi-Fi systems were also discussed, including dynamic frequency selection technology, duty cycle technology, and LBT technology. Our comprehensive survey on LTE-U will hopefully provide valuable references and guidelines for further indepth research in this area.

Acknowledgement: The authors thank the reviewers for their valuable suggestions, which greatly improved the quality of the paper. We thank LetPub (www.letpub.com) for its linguistic assistance during the preparation of this manuscript.

Funding Statement: This paper is supported by the research on the application of LTE-U in rail transit.

Conflicts of Interest: The authors declare that they have no conflicts of interest to report regarding the present study.

\section{References}

[1] B. Jo, M. J. Piran, D. Lee and D. Y. Suh, "Efficient computation offloading in mobile cloud computing for video streaming over 5G," Computers, Materials \& Continua, vol. 61, no. 2, pp. 439-463, 2019.

[2] M. Okhovvat and M. R. Kangavari, "A mathematical task dispatching model in wireless sensor actor networks," Computer Systems Science and Engineering, vol. 34, no. 1, pp. 5-12, 2019.

[3] Q. M. Chen, "Coexistence, convergence and optimization of LTE and Wi-Fi in the unlicensed spectrum," Ph.D. Dissertation, Zhejiang university, China, 2017.

[4] S. K. Zaman, M. A. T. Maqsood and K. Bilal, "A load balanced task scheduling heuristic for large-scale computing systems," Computer Systems Science and Engineering, vol. 34, no. 2, pp. 79-90, 2019.

[5] H. Hui, C. Zhou, S. Xu and F. Lin, "A novel secure data transmission scheme in industrial internet of things," China Communications, vol. 17, no. 1, pp. 73-88, 2020.

[6] Y. Y. Liu, "Research on coexistence mechanism of LTE-U and Wi-Fi in unlicensed bands," M.S. theses, Chongqing University of Posts and Telecommunications, China, 2018.

[7] S. Abeta, "Further advancements for E-UTRA physical layer aspects. USA, 3GPP technical specification TR, 36: V2," 2010. [Online]. Available: https://portal.3gpp.org/desktopmodules/Specifications/Specification.

[8] G. Dandachi, S. E. Elayoubi, T. Chahed and N. Chendeb, "Performance evaluation of user centric multihoming strategies in LTE/Wi-Fi networks," in Proc. IEEE Wireless Communications \& Networking Conf., Doha, Qatar, pp. 1-6, 2016.

[9] B. Chen, J. M. Chen and Y. Chen, "Coexistence of LTE-LAA and Wi-Fi on $5 \mathrm{GHz}$ with corresponding deployment scenarios: A survey,” IEEE Communications Surveys \&Tutorials, vol. 19, no. 1, pp. 7-32, 2017.

[10] S. Choi and S. Park, "Coexistence analysis of duty cycle method with Wi-Fi in unlicensed bands," in Proc. Int. Conf. on ICT Convergence, Jeju Island, Korea, pp. 894-897, 2015.

[11] Q. C. Zhao, S. Geirhofer, L. Tong and B. M. Sadler, "Optimal dynamic spectrum access via periodic channel sensing," in Proc. IEEE Wireless Communications \& Networking Conf., Kowloon, HK, China, pp. 33-37, 2007.

[12] H. U. Heng, M. Zheng and K. Yu, "Enhanced listen-before-talk scheme for LTE in unlicensed band," in Proc. IEEE Int. Conf. on Electronics Information and Emergency Communication, Beijing, BJ, China, pp. 168-173, 2016.

[13] Y. Liao and T. Y. Biank, "Decentralized dynamic spectrum access in full-duplex cognitive radio networks," in Proc. Int. Conf. on Communications, London, UK, pp. 7552-7557, 2015.

[14] S. Y. Lien, J. Lee and Y. C. Liang, "Random access or scheduling: Optimum LTE licensed assisted access unlicensed spectrum," IEEE Communications Letters, vol. 20, no. 3, pp. 590-593, 2016.

[15] J. Qiao, X. M. Shen and Y. J. He, "MAC-layer concurrent beamforming protocol for indoor millimeter-wave networks," IEEE Transactions on Vehicular Technology, vol. 64, no. 1, pp. 327-338, 2015. 
[16] M. Labib, V. Marojevic and J. H. Reed, "Extending LTE into the unlicensed spectrum: Technical analysis of the proposed variants," IEEE Communications Standards Magazine, vol. 1, no. 4, pp. 31-39, 2017.

[17] M. R. Khawer, J. Tang and F. Han, "ICIC-A proactive small cell interference mitigation strategy for improving spectral efficiency of LTE networks in the unlicensed spectrum," IEEE Transactions on Wireless Communications, vol. 15, no. 3, pp. 2303-2311, 2016.

[18] S. Sagari, S. Baysting, D. Saha, I. Seskar, W. Trappe et al., "Coordinated dynamic spectrum management of LTEU and Wi-Fi networks," in Proc. IEEE Int. Symp. on Dynamic Spectrum Access Networks, USA, pp. 209-220, 2015.

[19] N. Patriciello, S. Lagen, B. Bojovic and L. Giupponi, "NR-U and IEEE 802.11 technologies coexistence in unlicensed mmwave spectrum: Models and evaluation," IEEE Access, vol. 8, pp. 71254-71271, 2020.

[20] Nokia, "LTE for unlicensed spectrum. FRA," 2014. [Online]. Available: http://www.crplatform.nl/ Documentation/Docs/15/Nokia-LTE_unlicensed-whitepaper.pdf.

[21] Qualcomm, "MulteFire technology progress and benefits, and how it enables a new breed of neutral hosts, USA, qualcomm wireless evolution," 2016. [Online]. Available: https://www.qualcomm.com/videos/multefireprogress-and-benefits-and-how-it-enables-new-breed-neutral-hosts.

[22] J. Sun, J. Y. Oh, J. jeon and S. Ye, "Study on NR-based access to unlicensed spectrum (release 16). USA, 3GPP document TR 38.889 V1.0.0," 2018. [Online]. Available: https://portal.3gpp.org/desktopmodules/Specifications/ SpecificationDetails.aspx?specificationId=3235.

[23] S. Lagen, L. Giupponi, S. Goyal, N. Patriciello and B. Bojović, "New radio beam-based access to unlicensed spectrum: Design challenges and solutions," IEEE Communications Surveys \& Tutorials, vol. 22, no. 1, pp. 837, 2020.

[24] C. Rosa, M. Kuusela, F. Frederiksen and K. I. Pedersen, "Standalone LTE in unlicensed spectrum: Radio challenges, solutions, and performance of multeFire," IEEE Communications Magazine, vol. 56, no. 10, pp. 170-177, 2018.

[25] Qualcomm, "TE in unlicensed spectrum: Harmonious coexistence with Wi-Fi. USA, qualcomm wireless evolution," 2014. [Online]. Available: https:/www.qualcomm.com/media/documents/files/lte-unlicensedcoexistence-whitepaper.

[26] A. Babaei, J. Andreoli-Fang and B. Hamzeh, "On the impact of LTE-U on Wi-Fi performance," in Proc. IEEE Int. Symp. on Personal, Indoor and Mobile Radio Communications, Washington, DC, USA, pp. 1621-1625, 2014.

[27] Y. Mekonnen, M. Haque, I. Parvez, A. Moghadasi and A. Sarwat, "LTE and Wi-Fi coexistence in unlicensed spectrum with application to smart grid: A review," in Proc. IEEE/PES Transmission and Distribution Conf. and Exposition, Colorado, USA, pp. 1-5, 2018.

[28] J. Neto, S. Neto, P. Santana and A. Vicente, "Multi-cell LTE-U/Wi-Fi coexistence evaluation using a reinforcement learning framework," Sensors, vol. 20, no. 7, pp. 1855, 2020.

[29] T. Nihtila, V. Tykhomyrov, O. Alanen, M. A. Uusitalo, A. Sorri et al., "System performance of LTE and IEEE 802.11 coexisting on a shared frequency band," in Proc. IEEE Wireless Communications \& Networking Conf., Shanghai, China, pp. 1038-1043, 2013.

[30] A. Babaei, J. Andreoli-Fang and B. Hamzeh, "On the impact of LTE-U on Wi-Fi performance," in IEEE Int. Symp. on Personal, Indoor and Mobile Radio Communications, Washington, DC, USA, pp. 1621-1625, 2014.

[31] B. Chen, J. Chen and Y. Gao, "Coexistence of LTE-LAA and Wi-Fi on $5 \mathrm{GHz}$ with corresponding deployment scenarios: A survey," IEEE Communications Surveys \& Tutorials, vol. 19, no. 1, pp. 7-32, 2016.

[32] X. Jiang, M. Liu, C. Yang, Y. Liu and R. Wang, "A blockchain-based authentication protocol for WLAN mesh security access," Computers, Materials \& Continua, vol. 58, no. 1, pp. 45-59, 2019.

[33] A. Bhorkar, C. Ibars and P. Zong, "Performance analysis of LTE and Wi-Fi in unlicensed band using stochastic geometry," in Proc. IEEE Int. Symp. on Personal, Indoor and Mobile Radio Communications, Washington, DC, USA, pp. 1310-1314, 2014.

[34] S. Sagari, I. Seskar and D. Raychaudhuri, "Modeling the coexistence of LTE and Wi-Fi hetero geneous networks in dense deployment scenarios," in Proc. Int. Conf. on Communications, London, UK, pp. 2301-2306, 2015. 
[35] G. P. Villardi, C. S. Sum, C. Sun, Y. Alemseged, Z. Lair et al., "Efficiency of dynamic frequency selection based coexistence mechanisms for TV white space enabled cognitive wireless access points," IEEE Wireless Communications, vol. 19, no. 6, pp. 69-75, 2012.

[36] B. Jia and M. Tao, "A channel sensing based design for LTE in unlicensed bands," in Proc. Int. Conf. on Communications, London, UK, pp. 2332-2337, 2015.

[37] R. Ratasuk, M. A. Uusitalo, N. Mangalvedhe, A. Sorri, S. Iraji et al., "License-exempt LTE deployment in heterogeneous network," in Proc. IEEE Int. Symp. on Wireless Communication Systems, Paris, France, pp. 246-250, 2012.

[38] B. Xu, D. Huang and B. Mi, "Research on e-commerce transaction payment system basedf on c4.5 decision tree data mining algorithm," Computer Systems Science and Engineering, vol. 35, no. 2, pp. 113-121, 2020.

[39] Y. Song, K. W. Sung and Y. Han, "Coexistence of Wi-Fi and cellular with listen-before-talk in unlicensed spectrum," IEEE Communications Letters, vol. 20, no. 1, pp. 161-164, 2016.

[40] H. Ko, J. Lee and S. Pack, "A fair listen-before-talk algorithm for coexistence of LTE-U and WLAN," IEEE Transactions on Vehicular Technology, vol. 65, no. 12, pp. 10116-10120, 2016.

[41] R. Yin, G. Yu, A. Maaref and G. Li, "LBT based adaptive channel access for LTE-U systems," IEEE Transactions on Wireless Communications, vol. 15, no. 10, pp. 6585-6597, 2016.

[42] T. Tao, F. Han and Y. Liu, "Enhanced LBT algorithm for LTE-LAA in unlicensed band," in Proc. IEEE Int. Symp. on Personal, Indoor and Mobile Radio Communications, Hong Kong, China, pp. 1907-1911, 2015.

[43] B. X. Zheng, M. W. Wen, S. E. Lin, W. Wu, F. J. Chen et al., "Design of multi-carrier LBT for LAA\&WiFi coexistence in unlicensed spectrum," IEEE Network, vol. 34, no. 1, pp. 76-83, 2020.

[44] S. Han, Y. C. Liang, Q. Chen and B. H. Soong, "Licensed-assisted access for LTE in unlicensed spectrum: A MAC protocol design," IEEE Journal on Selected Areas in Communications, vol. 34, no. 10, pp. 2550-2561, 2016.

[45] Y. Li, J. Zheng and Q. Li, "Enhanced listen-before-talk scheme for frequency reuse of licensed assisted access using LTE," in Proc. IEEE Int. Symp. on Personal, Indoor and Mobile Radio Communications, Hong Kong, China, pp. 1918-1923, 2015.

[46] E. Almeida, A. Cavalcante, R. C. D. Paiva, F. Vieira, S. Choudhury et al., "Enabling LTE/Wi-Fi coexistence by LTE blank subframe allocation," in Proc. Int. Conf. on Communications, Sydney, AUS, pp. 5083-5088, 2013.

[47] Qualcomm, "LTE-U technology and coexistence, LTE-U forum workshop. USA, qualcomm wireless evolution," 2015. [Online]. Available: http://www. Iteuforum.org/workshop.html.

[48] Qualcomm, "Coexistence guidelines for LTE in unlicensed spectrum studies. USA, Wi-Fi alliance," 2015. [Online]. Available: https://www.qualcomm.com/media/documents/files/lte-unlicensed-coexistence-whitepaper.

[49] Qualcomm, "LTE-U Coexistence test plan. V0.8. USA, Wi-Fi alliance," 2016. [Online]. Available: https://www. wi-fi.org/news-events/newsroom/wi-fi-alliance-delivers-lte-u-coexistence-test-plan.

[50] N. Rupasinghe and I. Guven, "Reinforcement learning for licensed-assisted access of LTE in the unlicensed spectrum," in Proc. WCNC, New Orleans, LA, USA, pp. 1279-1284, 2015.

[51] C. Cano and D. J. Leith, "Coexistence of WiFi and LTE in unlicensed bands: proportional fair allocation scheme," in Proc. Int. Conf. on Communications, London, UK, pp. 2288-2293, 2015.

[52] J. Xiao and J. Zheng, "An adaptive channel access mechanism for LTE-U and Wi-Fi coexistence in an unlicensed spectrum," in Proc. Int. Conf. on Communications, London, UK, pp. 1-6, 2016.

[53] M. Cierny, H. Wang, R. Wichman, Z. Ding and C. Wijting, "On number of almost blank subframes in heterogeneous cellular networks," IEEE Transactions on Wireless Communications, vol. 12, no. 10, pp. 50615073, 2013.

[54] Y. N. Gu, Y. R. Zhang and L. X. Cai, "LTE-Unlicensed coexistence mechanism: A matching game framework," IEEE Wireless Communications, vol. 23, no. 6, pp. pp, 54-pp, 60, 2016.

[55] J. Lu and G. Fei, "Non-linear localization algorithm based on newton iterations," Journal of Internet of Things, vol. 2, no. 4, pp. 129-134, 2020. 